\title{
5-Hydroxyuracil Incision Activity Varies According to the Histological Grade of Non-muscle-invasive Bladder Cancer
}

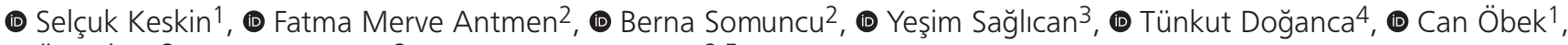 \\ ๑ Ümit Ince ${ }^{3}$, ๑ Ali Rıza Kural², @ Meltem Müftüoğlu2,5 \\ ${ }^{1}$ Acibadem Mehmet Ali Aydinlar University, School of Medicine, Department of Urology, Istanbul, Turkey \\ ${ }^{2}$ Acibadem Mehmet Ali Aydinlar University, Graduate School of Health Sciences, Department of Medical Biotechnology, Istanbul, Turkey \\ ${ }^{3}$ Acibadem Mehmet Ali Aydinlar University, School of Medicine, Department of Pathology, Istanbul, Turkey \\ ${ }^{4}$ Acibadem Taksim Hospital, Clinic of Urology, Istanbul, Turkey \\ ${ }^{5}$ Acibadem Mehmet Ali Aydinlar University, Faculty of Arts and Sciences, Department of Molecular Biology and Genetics, Istanbul, Turkey
}

\begin{abstract}
Objective: High levels of endonuclease III-like 1 (NTHL1) DNA glycosylase, which plays a role in the first step of the base excision repair pathway, has been related to cancer initiation and progression. 5-hydroxyuracil (5-OHU) oxidative base damage is a substrate for NTHL1 and endonuclease VIII-like 1 enzyme 1 (NEIL1) DNA glycosylases. This study investigates the association of 5-OHU incision activity with the risk of disease progression in patients with non-muscle-invasive bladder cancer (NMIBC) regarding grade and stage.

Materials and Methods: During transurethral resection of 17 NMIBC patients, the papillary tumour before monopolar resection and healthy bladder mucosal tissue from the same person were obtained using cold cup biopsy. Both the normal mucosa and NMIBC tumour were pathologically confirmed. The histological grade and stage were also determined. The 5-OHU incision activity of all tissues was measured using a radiolabelled 5-OHU modified base containing DNA substrate. Results: 5-OHU incision activity was significantly higher in all high-grade NMIBC tissue extracts compared with the corresponding normal tissues ( $p=0.001$ ). However, we found no significant difference in 5-OHU incision activity in low-grade NMIBC tissues ( $p=0.89$ ). There was also a significant increase in 5-OHU incision activity at the $\mathrm{Ta} / \mathrm{T} 1$ stage compared with the corresponding normal tissue $(\mathrm{p}=0.001)$.

Conclusion: The increase in 5-OHU incision activity according to the histological grade of NMIBC tissue indicates that this activity (mainly performed by NTHL1 and NEIL1 DNA glycosylases) might play a role in NMIBC prognosis. Thus, it could be used as a potential prognostic biomarker for NMIBC.

Keywords: Non-muscle-invasive bladder cancer, base excision repair, 5-hydroxyuracil incision, progression
\end{abstract}

\section{Introduction}

Bladder cancer is one of the most frequent (approximately 430,000 new cases per year) and most lethal (165,000 per year) cancers of the urinary system. Although its prevalence differs geographically, it is within the first five most prevalent and ten most lethal cancers (1). Bladder cancer is mainly grouped as nonmuscle-invasive bladder cancer (NMIBC) and muscle-invasive (MIBC). Around $80 \%$ of newly diagnosed cases are NMIBC $(2,3)$. Although bladder cancer's exact aetiology is unknown, environmental, genetic and epigenetic risk factors are thought to be related to the disease's aetiology and pathogenesis $(4,5)$. As an initial step in diagnosing and treating the disease, a complete transurethral resection (TUR) of the tumour is performed. In moderate and high-risk NMIBC patients, Bacillus CalmetteGuerin (BCG) installation remains the gold standard treatment regarding preventing disease recurrence and progression. However, approximately $40 \%$ of patients do not respond to BCG treatment $(6,7)$. Although conventional histopathological criteria, such as histological grade and stage, provide information to determine NMIBC progression and metastasis risk, patients with similar pathological features may have different disease courses $(3,8)$. This illustrates the importance of having an individualised therapeutic approach to bladder cancer. The present criteria are not adequate to define the risk factors for recurrence, progression and treatment response.

Recent preclinical and clinical cancer studies have shown that proteins involved in the base excision repair (BER) pathway

Cite this article as: Keskin S, Antmen FM, Somuncu B, Sağlıcan Y, Doğanca T, Öbek C, İnce Ü, Kural AR, Müftüoğlu M. 5-Hydroxyuracil Incision Activity Varies According to the Histological Grade of Non-muscle-invasive Bladder Cancer. Bull Urooncol 2021;20(1):45-48

Address for Correspondence: Meltem Müftüoğlu, Acibadem Mehmet Ali Aydinlar University, Graduate School of Health Sciences, Department of Medical Biotechnology, İstanbul, Turkey

Phone: +90 2165004131 E-mail: meltem.muftuoglu@acibadem.edu.tr ORCID-ID: orcid.org/0000-0001-5372-4780

Received: 25.01.2021 Accepted: 30.01.2021 
can be promising prognostic and predictive biomarkers and therapeutic targets to determine cancer risk, recurrence, progression and drug resistance. The BER pathway is the primary repair mechanism responsible for repairing oxidative base damages, monofunctional base modifications and single-strand breaks in DNA caused by environmental and endogenous agents. BER is a multistep pathway, and DNA damage-specific DNA glycosylase enzymes are involved in the first step of this pathway. DNA glycosylase enzymes recognise modified or damaged bases and form an apurinic/apyrimidinic (AP) site in the DNA chain by the cleavage of the $\mathrm{N}$-glycosidic bond. A single nucleotide gap is formed by the cleavage of the abasic AP site by AP endonuclease 1 (APE1), and the gap is filled by DNA polymerase $\beta$. The repair is completed by ligation of the nick $(9,10)$. Although DNA glycosylase enzymes are specific to DNA damage, they can also repair the same base damage. For example, the main DNA glycosylase that repairs 5-hydroxyuracil (5-OHU) base damage is the endonuclease III homologue (NTHL1). Endonuclease VIII-like 1 enzyme 1 (NEIL1) also uses this base damage as a substrate. 5-OHU base modification is the most common base modification resulting from oxidative deamination of cytosine by reactive oxygen species (11). 5-OHU DNA lesions are bypassed by DNA polymerases and pair with other bases during DNA replication, resulting in mutations. BER mechanism impairment causes mutagenic and/or cytotoxic DNA damage to accumulate in the genome and causes genomic instability. This situation is one of the main reasons for susceptibility to various cancers, such as bladder, lung and breast cancer. On the other hand, it has been shown that cancer cells have a very active BER mechanism that causes cancer cell viability, recurrence, progression, metastasis and resistance to genotoxic anticancer drugs $(10,11,12,13)$. For example, NTHL1 DNA glycosylase's overexpression has been associated with cancer progression and lymph node metastasis $(14,15,16)$. Therefore, this study investigated the association of 5-OHU activity with the risk of progression in patients with NMIBC using low-grade and high-grade NMIBC tissue and their corresponding normal tissues from the same person.

\section{Materials and Methods}

\section{NMIBC and Normal Bladder Tissue Samples from the Same Patient}

Before the TUR procedure, cold cup biopsies were acquired separately, first from healthy-appearing mucosa and then from cancer tissue in 17 newly diagnosed, treatment-naive bladder cancer patients at the Acibadem Maslak Hospital urology department. The samples were snap-frozen by immediately placing them in liquid nitrogen. A piece of the normal mucosa and cancer tissue were separated for conventional pathology to confirm the normal mucosa and diagnose and grade cancer cells (17). Ethical board approval was obtained from the Acibadem University medical ethics committee (ATADEK-2018/12). The informed consent form was filled in by all participants.

\section{Tissue Lysates Preparation of Normal and NMIBC Tissue}

Tissue lysates were prepared as previously described (18). Briefly, the tissue was homogenised by a Dounce glass homogeniser in cold whole-cell extraction buffer. The tissue homogenate was centrifuged at $16,000 \times \mathrm{g}$ at $4{ }^{\circ} \mathrm{C}$ for $15 \mathrm{~min}$. The pellet was dissolved, and tissue lysates were incubated at $4{ }^{\circ} \mathrm{C}$ for two hours in a shaking incubator. Then, they were centrifuged at $130,000 \times \mathrm{g}$ at $4{ }^{\circ} \mathrm{C}$ for one hour. The supernatant was dialysed at $4{ }^{\circ} \mathrm{C}$ overnight and centrifuged at $16,000 \times \mathrm{g}$ to remove the salts. The tissue lysates were kept at $-80{ }^{\circ} \mathrm{C}$. The tissue lysate protein content was measured with the Bradford protein assay at $595 \mathrm{~nm}$ using bovine serum albumin as a standard.

\section{Preparation of Radiolabelled 5-OHU Containing DNA Substrate}

The oligodeoxynucleotide sequences of DNA substrate containing 5-OHU base modification are as follows: $(X=5-\mathrm{OHU})$ 5'-GCTTAGCTTGGAATCGTA TCATGTAXACTCGT GTGCCGTGTA GACCGTGCC-3'; $3^{\prime}$-CGAATCGAACCTTAGCATAGTACATGTGAG CACACGGCA CATCTGGCACGG-5'.

The oligodeoxynucleotides were purchased from DNA Technology, Denmark. The 51 mer upper primer sequence of the substrate was labelled with $[\gamma-32 P] A T P$ using a polynucleotide kinase reaction protocol from the 5 -end (Perkin Elmer, USA). The annealing reaction was completed by incubating $[\gamma-32 \mathrm{P}]$ ATP labelled 51 mer primer and 51 mer template at $90{ }^{\circ} \mathrm{C}$ for 5 $\mathrm{min}$, and then slowly cooling to room temperature (18).

\section{5-OHU Incision Activity}

Incision of 5-OHU was performed in a reaction mixture containing $70 \mathrm{mM}$ HEPES-KOH, $\mathrm{pH}$ 7.4, $5 \mathrm{mM}$ EDTA, $1 \mathrm{mM}$ DTT, $50 \mathrm{mM} \mathrm{NaCl}, 10 \%$ glycerol and $50 \mathrm{fmol}$ of 32P-labelled double-stranded 5-OHU-containing the DNA substrate. The reactions were initiated by adding $0.5 \mu \mathrm{g}$ whole tissue extract and incubating at $37{ }^{\circ} \mathrm{C}$ for $30 \mathrm{~min}$. The reactions were stopped by adding formamide stop dye with $100 \mathrm{mM} \mathrm{NaOH}$. Samples were heated at $95{ }^{\circ} \mathrm{C}$ for $10 \mathrm{~min}$ and then run on 20\% PAGE-urea gel. Gels were visualised by Typhoon FLA 9500 Phosphorlmager (GE Healthcare, USA). The experiments were performed in triplicate, and the incision activity is presented as fmol of substrate converted to product per min per $\mu \mathrm{g}$ protein.

\section{Statistical Analysis}

Statistical analysis was performed using GraphPad Prism 6.1 software. Differences in NMIBC tissue activities and the same individual's corresponding normal tissue were analysed using the Wilcoxon matched-pairs signed-rank test. A $p<0.05$ was considered statistically significant. All statistical tests were two-sided.

\section{Results}

The mean age of the patients was $68.65 \pm 15.02$ years. A total of 6 patients had low-grade, and 11 patients had high-grade NMIBC. Of the patients, 14 had Ta stage and three of them had T1 disease. 5-OHU incision activity was measured as the incision of double-stranded oligodeoxynucleotide substrate containing 5-OHU at position 26. 5-OHU incision activity in all highgrade NMIBC tissue extracts was significantly higher than the corresponding normal tissues (Figure $1 \mathrm{~A}, \mathrm{p}=0.001$ ). In contrast, no statistically significant difference in the activity was observed in low-grade NMIBC tissues (Figure 1B, $\mathrm{p}=0.89$ ). The median of 
5-OHU incision was $0.20 \mathrm{fmol} / \mathrm{min} / \mu \mathrm{g}$ (range 0.15 to $0.44 \mathrm{fmol} /$ $\mathrm{min} / \mu \mathrm{g}$ ) in high-grade NMIBC tissues and it was $0.10 \mathrm{fmol} / \mathrm{min} /$ $\mu \mathrm{g}$ (range 0.06 to $0.17 \mathrm{fmol} / \mathrm{min} / \mu \mathrm{g}$ ) in their corresponding normal tissues. In low-grade NMIBC tissues, the median of the activity was $0.16 \mathrm{fmol} / \mathrm{min} / \mu \mathrm{g}$ (range 0.07 to 0.25 ) and it was $0.18 \mathrm{fmol} / \mathrm{min} / \mu \mathrm{g}$ (range 0.08 to $0.2 \mathrm{fmol} / \mathrm{min} / \mu \mathrm{g}$ ) in

A

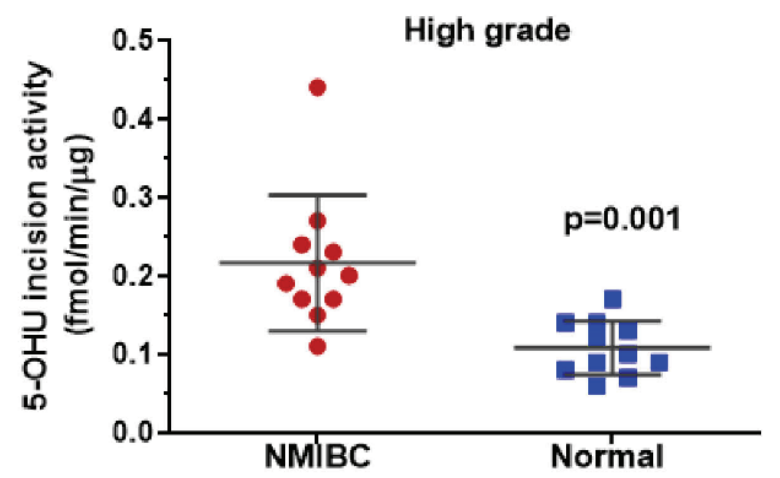

B

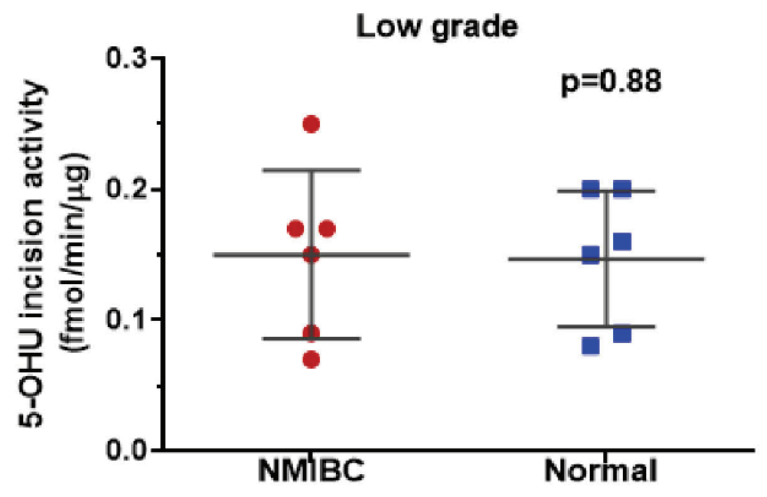

C

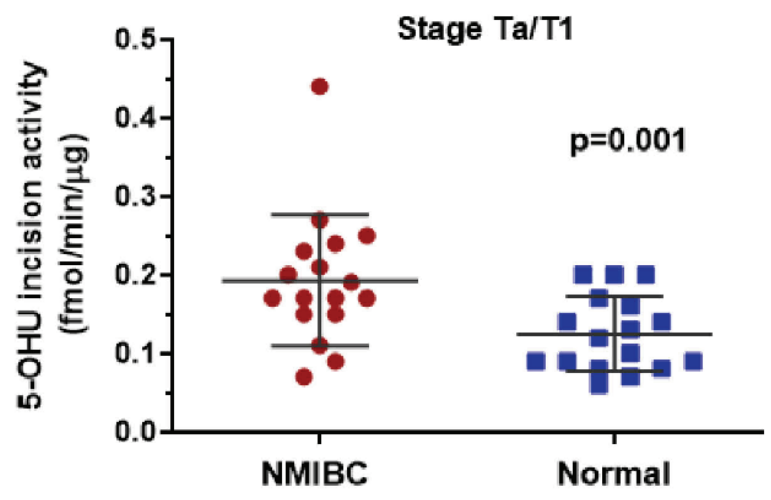

Figure 1. 5-OHU incision activity in NMIBC and their corresponding normal tissues. Quantitation of 5-OHU incision from high-grade (A), low-grade (B) and Ta/ T1 stages (C) of all NMIBC and their corresponding normal tissues ( $\mathrm{fmol} / \mu \mathrm{g} / \mathrm{min}$ ) 5-OHU: 5-hydroxyuracil, NMIBC: Non-muscle-invasive bladder cancer their corresponding normal tissues. NMIBC tissue at the Ta/T1 stage exhibited a significant increase in $5-\mathrm{OHU}$ activity (Figure $1 \mathrm{C}, \mathrm{p}=0.001$; median: $0.17 \mathrm{fmol} / \mathrm{min} / \mu \mathrm{g}$; range, 0.07 to 0.44 $\mathrm{fmol} / \mathrm{min} / \mu \mathrm{g}$ ) compared to the corresponding normal tissues (median: $0.12 \mathrm{fmol} / \mathrm{min} / \mu \mathrm{g}$; range, 0.06 to $0.2 \mathrm{fmol} / \mathrm{min} / \mu \mathrm{g}$ ). 5-OHU incision activity was 2.01 \pm 0.42 -fold higher in high-grade NMIBC tissue (Figure $1 \mathrm{~A}$ and Figure 2), whereas the activity was $0.97 \pm 0.2$-fold in low-grade NMIBC tissue (Figure 1B and Figure 2). Figure $2 C$ shows the inter-individual variation among NMIBC and normal tissue. 5-OHU incision activity is different in NMIBC and normal tissues, and each individual (Figure 2C).

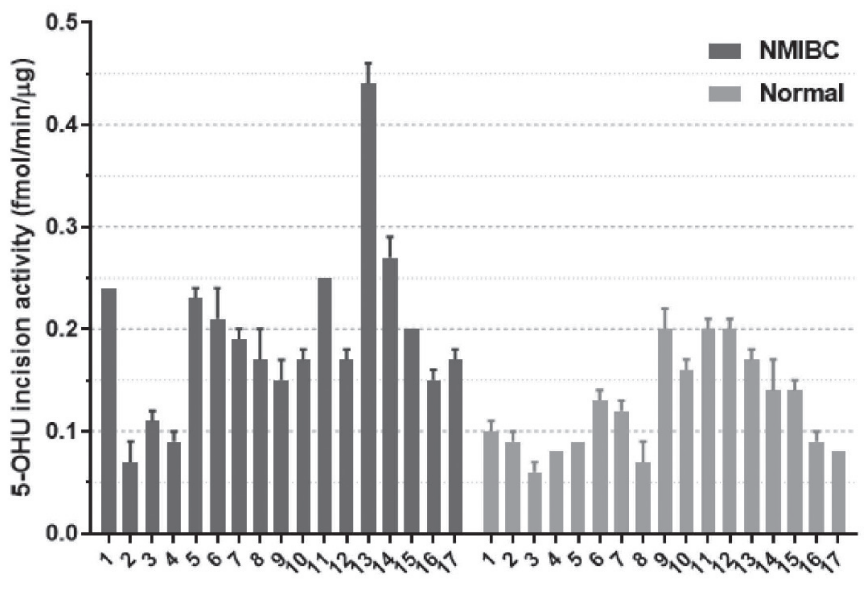

Figure 2. Inter-individual variability of 5-OHU incision activity in BER capacity of seventeen NMIBC and their corresponding normal tissues. Low-grade sample numbers are 2, 4, 9-12; high-grade sample numbers are 1, 3, 5-8, 13-17

5-OHU: 5-hydroxyuracil, NMIBC: Non-muscle-invasive bladder cancer, BER: Base excision repair

\section{Discussion}

In this study, it has been shown that $5-\mathrm{OHU}$ incision activity of high-grade NMIBC tissue is very high compared with that of healthy bladder tissue of the same individual. In contrast, the activity does not change in low-grade NMIBC tissues. Thus, the increased activity correlates with the grade of NMIBC tissues. 5-OHU is the substrate for both NTHL1 and NEIL1 DNA glycosylase enzymes (19), suggesting that these enzymes might play an important role in the progression and recurrence of NMIBC. Cells with high levels of NTHL1 and NEIL1 DNA glycosylase enzymes have been shown to protect themselves by preventing mutations induced by $5-\mathrm{OHU}$ modified base (16). Similarly, the aggressiveness and lymph node metastasis are related to increased levels of NTHL1 expression in colorectal cancers (15). In another study, high levels of NTHL1 gene expression were shown to cause genomic instability and tumour formation (14).

In NMIBC tissues, uracil DNA glycosylase activity, which repairs uracil base damage, was high. However, no difference was reported in 8-oxoguanine DNA glycosylase activity, which repairs 8-oxoguanine base damage. In the same study, total BER activity and APE1 and Pol $\beta$ activities of the BER pathway were increased in NMIBC tissues. These activities were statistically significant in both high-grade and low-grade NMIBC tissues than 
the corresponding normal tissues (18). A study comparing the expression levels of the APE1 gene in patients with high- and lowgrade NMIBC showed that APE1 gene expression was at a very high level in high-grade NMIBC tissue, and this increase was associated with the prognosis and recurrence of the disease $(20,21,22)$. In this study, the increase in 5-OHU incision activity depended on the degree of NMIBC, thereby demonstrating that this activity can also be used as a prognostic biomarker for NMIBC.

Every individual has a different DNA repair capacity. The emergence of BER capacity differences between individuals and even between normal and cancer tissues helps to personalise cancer treatment by enabling the individual to understand their sensitivity to environmental toxins and their response to certain chemotherapeutic agents (23). Determining each NMIBC patient's BER capacity will facilitate their selected treatment approach that will provide the maximum benefit for NMIBC patients and predict the cancer cells' response to treatment.

\section{Study Limitations}

The limitation of this study was its small sample size. The number of patients could be increased for future biomarker studies.

\section{Conclusion}

In this study, the increase in 5-OHU incision activity in highgrade NMIBC tissues was determined and associated with NMIBC tissue grade. This activity and DNA glycosylase enzymes using a 5-OHU modified base as a substrate (mainly NTHL1 and NEIL1) might play a role in the progression of NMIBC. It could be used as a potential prognostic biomarker for NMIBC.

\section{Acknowledgements}

Publication: The results of the study were not published in full or in part in form of abstracts.

Contribution: There is not any contributors who may not be listed as authors.

Conflict of Interest: No conflict of interest was declared by the authors.

Financial Disclosure: The authors declared that this study received no financial support.

\section{Ethics}

Ethics Committee Approval: Ethical board approval was obtained from the Acibadem University medical ethics committee (ATADEK-2018/12).

Informed Consent: The informed consent form was filled in by all participants.

Peer-review: Externally and internally peer-reviewed.

\section{Authorship Contributions}

Concept: S.K., M.M., Design: S.K., C.Ö., Ü.İ., A.R.K., M.M., Data Collection or Processing: S.K., F.M.A., B.S., Y.S., T.D., C.Ö., Ü.I.,, A.R.K., M.M., Analysis or Interpretation: S.K., F.M.A., B.S., Y.S., Ü.I., A.R.K., M.M., Literature Search: A.A., Ç.D., A.E., Writing: M.M., S.K.

\section{References}

1. Antoni S, Ferlay J, Soerjomataram I, et al. Bladder Cancer Incidence and Mortality: A Global Overview and Recent Trends. Eur Urol 2017;71:96-108.

2. Inamura K. Bladder Cancer: New Insights into Its Molecular Pathology. Cancers (Basel) 2018;10:100.

3. van Rhijn BW, Burger M, Lotan $\mathrm{Y}$, et al. Recurrence and progression of disease in non-muscle-invasive bladder cancer: from epidemiology to treatment strategy. Eur Urol 2009;56:430-442.

4. Letasiova S, Medve'ova A, Sovcikova A, et al. Bladder cancer, a review of the environmental risk factors. Environ Health 2012;11 Suppl 1(Suppl 1):S11.

5. Pelucchi C, Bosetti C, Negri E, et al. Mechanisms of disease: The epidemiology of bladder cancer. Nat Clin Pract Urol 2006;3:327-340.

6. Rayn KN, Hale GR, Grave GP, Agarwal PK. New therapies in nonmuscle invasive bladder cancer treatment. Indian J Urol 2018;34:11-19.

7. Packiam VT, Johnson SC, Steinberg GD. Non-muscle-invasive bladder cancer: Intravesical treatments beyond Bacille Calmette-Guerin. Cancer 2017;123:390-400.

8. Knowles MA, Hurst CD. Molecular biology of bladder cancer: new insights into pathogenesis and clinical diversity. Nat Rev Cancer 2015;15:25-41.

9. Kelley MR, Logsdon D, Fishel ML. Targeting DNA repair pathways for cancer treatment: what's new? Future Oncol 2014;10:1215-1237.

10. Illuzzi JL, Wilson DM, 3rd. Base excision repair: contribution to tumorigenesis and target in anticancer treatment paradigms. Curr Med Chem 2012;19:3922-3936.

11. Brenerman BM, Illuzzi JL, Wilson DM 3rd. Base excision repair capacity in informing healthspan. Carcinogenesis 2014;35:2643-2652.

12. Wallace SS. Base excision repair: a critical player in many games. DNA Repair (Amst) 2014;19:14-26.

13. Sarasin A, Kauffmann A. Overexpression of DNA repair genes is associated with metastasis: a new hypothesis. Mutat Res 2008;659:49-55.

14. Limpose KL, Trego KS, Li Z, et al. Overexpression of the base excision repair NTHL1 glycosylase causes genomic instability and early cellular hallmarks of cancer. Nucleic Acids Res. 2018;46:4515-4532.

15. Koketsu S, Watanabe T, Nagawa H. Expression of DNA repair protein: $\mathrm{MYH}, \mathrm{NTH} 1$, and MTH1 in colorectal cancer. Hepatogastroenterology 2004;51:638-642.

16. Shinmura K, Kato H, Kawanishi $Y$, et al. Defective repair capacity of variant proteins of the DNA glycosylase NTHL1 for 5-hydroxyuracil, an oxidation product of cytosine. Free Radic Biol Med 2019;131:264-273.

17. Stenzl A, Cowan NC, De Santis M, et al. The updated EAU guidelines on muscle-invasive and metastatic bladder cancer. Eur Urol 2009;55:815-825.

18. Somuncu B, Keskin S, Antmen FM, et al. Non-muscle invasive bladder cancer tissues have increased base excision repair capacity. Sci Rep 2020;10:16371.

19. Katafuchi A, Nakano T, Masaoka A, et al. Differential Specificity of Human and Escherichia coli Endonuclease III and VIII Homologues for Oxidative Base Lesions. J Biol Chem 2004;279:14464-14471.

20. Choi S, Shin JH, Lee YR, et al. Urinary APE1/Ref-1: A Potential Bladder Cancer Biomarker. Dis Markers 2016;2016:7276502.

21. Shin JH, Choi S, Lee YR, et al. APE1/Ref-1 as a Serological Biomarker for the Detection of Bladder Cancer. Cancer Res Treat 2015;47:823-833.

22. Chantre-Justino M, Alves $G$, Britto $C$, et al. Impact of reduced levels of APE1 transcripts on the survival of patients with urothelial carcinoma of the bladder. Oncol Rep 2015;34:1667-1674.

23. Wilson DM 3rd, Kim D, Berquist BR, Sigurdson AJ. Variation in base excision repair capacity. Mutat Res 2011;711:100-112. 